\title{
Gravitational self-force correction to the innermost stable circular orbit of a Schwarzschild black hole
}

\author{
Leor Barack ${ }^{1}$ and Norichika Sago ${ }^{1,2}$ \\ ${ }^{1}$ School of Mathematics, University of Southampton, Southampton, SO17 1BJ, United Kingdom \\ ${ }^{2}$ Yukawa Institute for Theoretical Physics, Kyoto University, Kyoto 606-8502, Japan
}

(Dated: October 22, 2018)

\begin{abstract}
The innermost stable circular orbit (ISCO) of a test particle around a Schwarzschild black hole of mass $M$ has (areal) radius $r_{\text {isco }}=6 M G / c^{2}$. If the particle is endowed with mass $\mu(\ll M)$, it experiences a gravitational self-force whose conservative piece alters the location of the ISCO. Here we calculate the resulting shifts $\Delta r_{\text {isco }}$ and $\Delta \Omega_{\text {isco }}$ in the ISCO's radius and frequency, at leading order in the mass ratio $\mu / M$. We obtain, in the Lorenz gauge, $\Delta r_{\text {isco }}=-3.269( \pm 0.003) \mu G / c^{2}$ and $\Delta \Omega_{\text {isco }} / \Omega_{\text {isco }}=0.4870( \pm 0.0006) \mu / M$. We discuss the implications of our result within the context of the extreme-mass-ratio binary inspiral problem.

PACS numbers: 04.25.Nx, 04.25.dg, 04.70.Bw
\end{abstract}

The last few years have seen a breakthrough in the development of computational tools for tackling the two-body problem in general relativity [1]. Numericalrelativistic (NR) codes can now track the complicated nonlinear dynamics of a spacetime describing the inspiral and merger of two black holes with comparable masses. A subset of problems which remains so far beyond the reach of full NR treatment concerns the radiative inspiral of a small compact object into a black hole of a much larger mass. (The study of such extreme-mass-ratio inspirals is motivated strongly by the prospects of observing gravitational waves from captures of compact stars by massive black holes in galactic nuclei - a particularly exciting source for the planned space-based gravitational wave detector LISA [2].) The large span of lengthscales and long inspiral timescale render this problem intractable using current NR technology [3].

The problem, however, falls naturally within the remit of black-hole perturbation theory, which utilizes the small mass ratio $\eta$ as an expansion parameter. In this approach, the small object is treated as a structureless point particle, which moves on the fixed background of the massive hole. At the test-mass limit $(\eta \rightarrow 0)$ the particle traces a geodesic of the black hole geometry. When $\eta$ is finite (yet small), the particle interacts with its own gravitational field, giving rise to a gravitational self force (SF). The SF has a dissipative piece which drives the radiative inspiral; it also has a conservative component which affects the evolution of the orbital phases.

How to regularize the gravitational self-interaction in curved spacetime is now a well understood problem in fundamental relativity [4], and a program to calculate the gravitational SF in the strong-field exterior of a Kerr black hole is well underway. The first actual quantitative results were obtained for radial plunge [5] and circular orbits [6] in a Schwarzschild exterior. In the circular orbit case, accurate computations of conservative SF effects were performed using two independent methods, successfully tested against each other and against results from post-Newtonian (PN) theory [7, 8]. The authors of this letter have recently completed the development of a com- putational platform for calculations of the SF for generic (bound, eccentric) orbits in Schwarzschild. Details of this work, which we consider a significant milestone in the SF program, are given in a forthcoming paper [9].

As a first application of our code, we report here the values of the $O(\eta)$ shifts in the location and frequency of the innermost stable circular orbit (ISCO) of a Schwarzschild black hole due to the conservative piece of the gravitational SF. This represents a first concrete calculation of a conservative SF effect with a clear physical interpretation and (potentially) observable consequences. The value of the ISCO frequency, which is gauge-invariant (within a class of physically-reasonable gauges - see below), has been utilized in the past as a convenient reference for comparison and calibration of PN schemes [10]. Our result is exact at $O(\eta)$ (within a controlled numerical error), and we expect it to provide an accurate benchmark against which future PN calculations (and potentially also future NR results for binaries with small $\eta$ ) could be tested.

The radiative decay of a physical circular orbit proceeds through an "adiabatic" inspiral epoch, during which gravitational radiation reaction slowly removes energy and angular momentum from the system, on to a brief plunge episode in which the particle drops into the black hole along a nearly-geodesic trajectory. The transition from inspiral to plunge occurs at radii localized around the ISCO. The properties of the transition regime were studied by Ori and Thorne (OT) [11] (for circular equatorial orbits in Kerr), with later generalizations by O'Shaughnessy [12] and Sundararajan [13]. OT showed, in particular, that the frequency bandwidth of the radiative transition scales as $\sim \eta^{2 / 5}$ : The transition becomes more "abrupt" - less gradual - with smaller $\eta$. OT (as also [12, 13]) did not tackle the local SF directly. Instead, they relied on (numerical) computations of the flux of energy in the radiated gravitational waves, from which the dissipative piece of the SF was inferred indirectly. OT had to ignore the conservative piece of the SF, yet unknown at the time of their analysis. Our work is complementary to OT's in that we consider only conser- 
vative effects and ignore dissipation: it is only then that the ISCO becomes precisely localizable. As we shall conclude below, our result justifies a posteriori the omission of conservative SF terms in the OT analysis (at least in the special Schwarzschild case).

In what follows we (i) derive formulae for the conservative $O(\eta)$ shift in the ISCO radius and frequency in terms of the conservative piece of the SF; (ii) describe in brief the numerical method used for computing the conservative SF (delegating details to a forthcoming paper [9]); and (iii) state our results and discuss their implications. In the rest of this letter we use standard geometrized units, with $G=c=1$. We denote by $M$ the mass of the background Schwarzschild black hole and by $\mu$ the mass of the particle (hence $\mu / M=\eta$ ). The particle's orbit is described in standard Schwarzschild coordinates by $t=t_{\mathrm{p}}(\tau), r=r_{\mathrm{p}}(\tau)$ and $\varphi=\varphi_{\mathrm{p}}(\tau)$, where $\tau$ is proper time; without loss of generality we take $\theta_{\mathrm{p}}=\pi / 2$. The metric signature is -+++ , and tensorial indices are raised and lowered using the background metric.

Formula for the ISCO shift:- At the limit $\mu \rightarrow 0$ the particle traces a timelike geodesic of the Schwarzschild geometry, described (using overhats to distinguish the geodesic from the perturbed orbit discussed later) by

$$
\begin{gathered}
\dot{\hat{r}}_{\mathrm{p}}^{2}=E^{2}-V_{\mathrm{eff}}\left(\hat{r}_{\mathrm{p}}, L^{2}\right), \\
\dot{\hat{t}}_{\mathrm{p}}=E / f\left(\hat{r}_{\mathrm{p}}\right), \quad \dot{\hat{\varphi}}_{\mathrm{p}}=L / \hat{r}_{\mathrm{p}}^{2} .
\end{gathered}
$$

Here an overdot denotes $d / d \hat{\tau}, E$ and $L$ are, respectively, the specific energy and angular momentum (constants of the motion), $f(r)=1-2 M / r$, and the radial effective potential is $V_{\text {eff }}\left(r, L^{2}\right)=f\left(1+L^{2} / r^{2}\right)$. Bound orbits exist only for $L>\sqrt{12} M$, in which case $V_{\text {eff }}$ has two extremum points and $E^{2}-V_{\text {eff }}$ can have 3 distinct roots. In the latter case, the radial motion is bounded as $r_{\min } \leq \hat{r}_{\mathrm{p}}(\hat{\tau}) \leq r_{\max }$, where $r_{\min }$ ("periapsis") and $r_{\max }$ ("apoapsis") are the second-largest and largest roots, respectively. For such orbits we define the "eccentricity" $e$ and "semi-latus rectum" $r_{0}$ through

$$
r_{\min }=r_{0} /(1+e), \quad r_{\max }=r_{0} /(1-e) .
$$

The orbits are fully parameterized (up to initial conditions) by any of the pairs $\{E, L\},\left\{r_{\max }, r_{\min }\right\}$, or $\left\{e, r_{0}\right\}$. Stable circular orbits occur when $E^{2}$ equals the minimum of $V_{\text {eff }}$; they have $e=0$ and radius $r_{\min }=r_{\max }=r_{0}=$ $\left(L^{2}+L \sqrt{L^{2}-12 M^{2}}\right) /(2 M)$. No bound orbits exist for $L<\sqrt{12} M$. The circular orbit with $L=\sqrt{12} M$ is the ISCO; it has radius $r_{0}=6 \mathrm{M}$.

The nature of the ISCO as a separatrix for stable circular orbits is understood from the following dynamical consideration. Still working at $\mu \rightarrow 0$, consider a small-e perturbation of a circular geodesic with radius $r_{0}$, such that the resulting slightly-eccentric geodesic has turning points $r_{\min }$ and $r_{\max }$ as in Eq. (3). For this orbit we write

$$
\hat{r}_{\mathrm{p}}(\hat{\tau})=r_{0}+e \hat{r}_{1}(\hat{\tau})+O\left(e^{2}\right),
$$

where $\hat{r}_{1}(\hat{\tau})$ is to be determined below. The $r$ component of the test-particle's geodesic equation reads

$$
\ddot{\hat{r}}_{\mathrm{p}}=-\frac{1}{2} \frac{\partial V_{\text {eff }}}{\partial \hat{r}_{\mathrm{p}}} \equiv \mathcal{F}_{\text {eff }}\left(\hat{r}_{\mathrm{p}}, L^{2}\right),
$$

$\mathcal{F}_{\text {eff }}$ being an effective radial force (per unit test mass) for the geodesic motion. The linear variation of $\mathcal{F}_{\text {eff }}$ with respect to $e$ (with fixed $r_{0}$ and $\hat{\tau}$ ) is $\delta_{e} \mathcal{F}_{\text {eff }}=$ $\left(\partial \mathcal{F}_{\text {eff }} / \partial \hat{r}_{\mathrm{p}}\right) \delta_{e} \hat{r}_{\mathrm{p}}+\left(\partial \mathcal{F}_{\text {eff }} / \partial L\right) \delta_{e} L$, where the partial derivatives are evaluated at $e=0$, and where $\delta_{e} \hat{r}_{\mathrm{p}}=e \hat{r}_{1}$. $\delta_{e} L$ is determined by solving $E^{2}=V_{\mathrm{eff}}\left(r_{\mathrm{min}}, L^{2}\right)$ and $E^{2}=V_{\mathrm{eff}}\left(r_{\max }, L^{2}\right)$ simultaneously for $E$ and $L$, and then using Eqs. (3); this gives $L^{2}=M r_{0}^{2} /\left(r_{0}-3 M-M e^{2}\right)$ and hence $\delta_{e} L=0$. At $O(e)$, therefore, Eq. (5) reads

$$
\ddot{\hat{r}}_{1}(\hat{\tau})=-\hat{\omega}_{r}^{2} \hat{r}_{1}(\hat{\tau}),
$$

with

$$
\hat{\omega}_{r}^{2}=-\left.\frac{\partial \mathcal{F}_{\mathrm{eff}}}{\partial \hat{r}_{\mathrm{p}}}\right|_{\hat{r}_{\mathrm{p}}=r_{0}}=\frac{M\left(r_{0}-6 M\right)}{r_{0}^{3}\left(r_{0}-3 M\right)} .
$$

Thus, the $O(e)$ radial motion is simple-harmonic with frequency $\hat{\omega}_{r}$. The orbit is stable under small-e perturbations when $\hat{\omega}_{r}^{2}>0$, namely for $r_{0}>6 M$ (no circular timelike geodesics exist for $\left.r_{0} \leq 3 M\right)$. The condition $\hat{\omega}_{r}=0$ identifies the ISCO at $r_{0}=6 M$.

We now turn to consider the $O(\mu)$ conservative correction to the orbit. The physical SF along the geodesic $\hat{r}_{\mathrm{p}}(\hat{\tau})$ is constructed (as prescribed in [4]) from the retarded metric perturbation associated with the particle. We denote this SF by $\mu F_{\text {ret }}^{\alpha}$, and further denote by $\mu F_{\text {adv }}^{\alpha}$ the force derived in just the same way from the advanced perturbation [we factor out $\mu$ for later convenience, noting our F's $(\propto \mu)$ describe self acceleration]. The conservative piece of the SF (per unit $\mu$ ) is defined through $F^{\alpha} \equiv\left(F_{\text {ret }}^{\alpha}+F_{\text {adv }}^{\alpha}\right) / 2$. It is convenient to take $\hat{\tau}=0$ at a periapsis of $\hat{r}_{\mathrm{p}}(\hat{\tau})$, in which case we have the symmetry relation [14] $F_{\text {adv }}^{\alpha}(\hat{\tau})=\epsilon_{\alpha} F_{\text {ret }}^{\alpha}(-\hat{\tau})$ (no summation over $\alpha$ ), where $\epsilon_{\alpha}=(-1,1,1,-1)$ in Schwarzschild coordinates. This gives us a practical formula for extracting the conservative piece from the full (retarded) SF:

$$
F^{\alpha}(\hat{\tau})=\left[F_{\text {ret }}^{\alpha}(\hat{\tau})+\epsilon_{\alpha} F_{\text {ret }}^{\alpha}(-\hat{\tau})\right] / 2 .
$$

Notice that, since both $F^{r}$ and $\hat{r}_{\mathrm{p}}$ are periodic and even in $\hat{\tau}$ [and $\hat{r}_{\mathrm{p}}(\hat{\tau})$ is monotonic on $0 \leq \hat{\tau} \leq \pi / \hat{\omega}_{r}$ ], we may express $F^{r}$ as a function of $\hat{r}_{\mathrm{p}}$ only (for given $r_{0}, e$ ). We write $F^{r}=F^{r}\left(\hat{r}_{\mathrm{p}} ; r_{0}, e\right)$.

Under the effect of $F^{\alpha}$, the nontrivial components of the equation of motion read

$$
\begin{aligned}
& \ddot{r}_{\mathrm{p}}=\mathcal{F}_{\text {eff }}\left(r_{\mathrm{p}}, L^{2}\right)+F^{r}, \\
& \dot{E}=-F_{t}, \quad \dot{L}=F_{\varphi},
\end{aligned}
$$

where $E \equiv \dot{t}_{\mathrm{p}} / f\left(r_{\mathrm{p}}\right)$ and $L \equiv \dot{\varphi}_{\mathrm{p}} / r_{\mathrm{p}}^{2}$ are (in general) no longer constant along the orbit, and hereafter an over$\operatorname{dot}$ denotes $d / d \tau$. From symmetry, the perturbed orbit 
remains equatorial. We assume $r_{\mathrm{p}}(\tau)$ remains bounded between some $r_{\min }$ and $r_{\max }$, define $r_{0}$ and $e$ as in Eq. (3), and take $\tau=0$ at a periapsis. Then we may write, through $O(\mu), F^{r}=F^{r}\left(r_{\mathrm{p}} ; r_{0}, e\right)$. By virtue of Eqs. (8) and (10) we similarly have $E=E\left(r_{\mathrm{p}} ; r_{0}, e\right)$ and $L=L\left(r_{\mathrm{p}} ; r_{0}, e\right)$ through $O(\mu)$.

Consider first circular orbit solutions of the set (910). In this case $E$ and $L$ are constant along the orbit. Their values are obtained by solving Eqs. (11) (with $\hat{r}_{\mathrm{p}} \rightarrow r_{\mathrm{p}}$ ) and (9) simultaneously, with $\dot{r}_{\mathrm{p}}=\ddot{r}_{\mathrm{p}}=0$ and $r_{\mathrm{p}}=r_{0}$. This gives

$$
\begin{aligned}
E_{0}^{2} & =r_{0} f_{0}^{2}\left(r_{0}-3 M\right)^{-1}\left[1-\left(r_{0} / f_{0}\right) F_{0}^{r}\right], \\
L_{0}^{2} & =M r_{0}^{2}\left(r_{0}-3 M\right)^{-1}\left[1-\left(r_{0}^{2} / M\right) F_{0}^{r}\right],
\end{aligned}
$$

where subscripts ' 0 ' denote circular-orbit values.

To identify the new location of the ISCO we again consider a small-e perturbation of the circular orbit. Writing $r_{\mathrm{p}}(\tau)$ as in Eq. (4), we find that $r_{1}(\tau)$ again satisfies an equation of the form (6), where the frequency is now

$$
\omega_{r}^{2}=-\frac{d}{d r_{\mathrm{p}}}\left[\mathcal{F}_{\mathrm{eff}}\left(r_{\mathrm{p}}, L^{2}\left(r_{\mathrm{p}}\right)\right)+F^{r}\left(r_{\mathrm{p}}\right)\right]_{r_{\mathrm{p}}=r_{0}} .
$$

In obtaining this result we have replaced the linear variations $\delta_{e} L$ and $\delta_{e} F^{r}$ with $r_{1} \delta_{r_{\mathrm{p}}} L$ and $r_{1} \delta_{r_{\mathrm{p}}} F^{r}$, respectively, making use of the fact that, through $O(e), L$ and $F^{r}$ depend on $e$ only through $r_{\mathrm{p}}\left(\tau ; r_{0}, e\right)$. That this is true is shown as follows. Let us formally expand $L=L\left(r_{\mathrm{p}} ; r_{0}, e\right)=L^{(0)}\left(r_{\mathrm{p}} ; r_{0}\right)+e L^{(1)}\left(r_{\mathrm{p}} ; r_{0}\right)+O\left(e^{2}\right)$, where the coefficients $L^{(n)}$ depend on $e$ only through $r_{\mathrm{p}}$, and $O\left(e^{2}\right)$ represents terms whose explicit dependence on $e$ is at least quadratic. Now note from Eq. (3) that formally replacing $e \rightarrow-e$ is equivalent to replacing $r_{\min } \leftrightarrow r_{\max }$; hence $L\left(r_{\min } ; r_{0}, \mp e\right)=L\left(r_{\max } ; r_{0}, \pm e\right)$, giving $L^{(1)}\left(r_{\min } ; r_{0}\right)=-L^{(1)}\left(r_{\max } ; r_{0}\right)$. Assuming $L$ is a continuous function of $r_{\mathrm{p}}$, the last result implies that $L^{(1)}=O(e)$ for all $r_{\mathrm{p}}$. We thus have $L=L^{(0)}\left(r_{\mathrm{p}} ; r_{0}\right)+$ $O\left(e^{2}\right)$, with the conclusion that, working at $O(e), L$ may depend on $e$ only through $r_{\mathrm{p}}$. The same argument - with the same conclusion - applies to $F^{r}$.

It is now useful to describe the $O(e)$ orbit more explicitly: Assuming periapsis at $\tau=0$, Eq. (6) (overhats removed) integrates to give $r_{1}=-r_{0} \cos \omega_{r} \tau$, hence

$$
r_{\mathrm{p}}(\tau)=r_{0}\left(1-e \cos \omega_{r} \tau\right)+O\left(e^{2}\right) .
$$

We can then expand $L\left(r_{\mathrm{p}}\right)$ through $O(e)$ in the form $L=L_{0}+e r_{1} L^{\prime}\left(r_{0}\right)=L_{0}-e r_{0} L^{\prime}\left(r_{0}\right) \cos \omega_{r} \tau$, where a prime denotes $d / d r_{\mathrm{p}}$ and $L_{0}$ is the circular-orbit value, given in Eq. (12). Since $F_{\varphi}=\dot{L}$, we obtain the form

$$
F_{\varphi}=e \omega_{r} F_{\varphi}^{1} \sin \omega_{r} \tau+O\left(e^{2}\right),
$$

where $F_{\varphi}^{1} \equiv r_{0} L^{\prime}\left(r_{0}\right)$. Similarly, we expand $F^{r}\left(r_{\mathrm{p}}\right)=$ $F_{0}^{r}+e r_{1} F^{r \prime}\left(r_{0}\right)+O\left(e^{2}\right)$, giving

$$
F^{r}=F_{0}^{r}+e F_{1}^{r} \cos \omega_{r} \tau+O\left(e^{2}\right),
$$

where $F_{1}^{r} \equiv-r_{0} F^{r^{\prime}}\left(r_{0}\right)$. Replacing $L^{\prime}\left(r_{0}\right) \rightarrow F_{\varphi}^{1} / r_{0}$ and $F^{r^{\prime}}\left(r_{0}\right) \rightarrow-F_{1}^{r} / r_{0}$ in Eq. (13) and substituting for $L_{0}$ from Eq. (12), we finally obtain

$$
\omega_{r}^{2}=\hat{\omega}_{r}^{2}+\alpha\left(r_{0}\right) F_{0}^{r}+\beta\left(r_{0}\right) F_{1}^{r}+\gamma\left(r_{0}\right) F_{\varphi}^{1},
$$

where $\alpha=-3 r_{0}^{-1}\left(r_{0}-4 M\right) /\left(r_{0}-3 M\right), \beta=r_{0}^{-1}$ and $\gamma=$ $-2 r_{0}^{-4}\left[M\left(r_{0}-3 M\right)\right]^{1 / 2}$. This formula describes the $O(\mu)$ conservative shift in the radial frequency off its geodesic value. Note it requires knowledge of the SF through $O(e)$.

The perturbed ISCO radius $r_{\text {isco }}$ is now obtained from the condition $\omega_{r}^{2}\left(r_{\text {isco }}\right)=0$. Recalling Eqs. (7) this gives

$$
\begin{aligned}
\Delta r_{\text {isco }} & \equiv r_{\text {isco }}-6 M \\
& =\left.\left(r_{0}^{3} / M\right)\left(3 M-r_{0}\right)\left(\alpha F_{0}^{r}+\beta F_{1}^{r}+\gamma F_{\varphi}^{1}\right)\right|_{r_{0}=6 M} \\
& =216 M^{2} F_{0 \text { is }}^{r}-108 M^{2} F_{1 \text { is }}^{r}+\sqrt{3} F_{\varphi \text { is }}^{1},
\end{aligned}
$$

where in the second line we were allowed to replace $r_{\text {isco }} \rightarrow 6 M$ since the SF terms are already $O(\mu)$ [so the error introduced affects $\Delta r_{\text {isco }}$ only at $\left.O\left(\mu^{2}\right)\right]$. We have denoted $F_{0 \text { is }}^{r} \equiv F_{0}^{r}\left(r_{0}=6 M\right)$ and similarly for $F_{1 \text { is }}^{r}, F_{\varphi \text { is }}^{1}$. Note $\Delta r_{\text {isco }}$ is gauge dependent, just like the SF itself [15].

A more physically-meaningful quantity associated with the circular orbit is the azimuthal frequency $\Omega \equiv$ $d \varphi_{\mathrm{p}} / d t=\dot{\varphi}_{\mathrm{p}} / \dot{t}_{\mathrm{p}}$. As discussed in [8], $\Omega$ is invariant under all $O(\mu)$ gauge transformations whose generators respect the helical symmetry of the circular-orbit configuration [16]. An expression for the SF-corrected $\Omega$ is obtained using Eqs. (11) and (12):

$$
\Omega=\hat{\Omega}\left[1-\frac{r_{0}\left(r_{0}-3 M\right)}{2 M f_{0}} F_{0}^{r}\right],
$$

where $\hat{\Omega} \equiv\left(M / r_{0}^{3}\right)^{1 / 2}$ is the geodesic (no $\mathrm{SF}$ ) value. Evaluated at $r_{0}=r_{\text {isco }}$, the SF-induced frequency shift $\Delta \Omega \equiv \Omega-\hat{\Omega}$ reads [through $O(\mu)$ ]

$$
\Delta \Omega_{\text {isco }}=-\Omega_{\text {isco }}\left[\frac{1}{4} \Delta r_{\text {isco }} / M+\frac{27}{2} M F_{0 \text { is }}^{r}\right],
$$

where $\Omega_{\text {isco }} \equiv \hat{\Omega}\left(r_{0}=6 M\right)=\left(6^{3 / 2} M\right)^{-1}$. Equations analogous to (18) and (20) were obtained by Diaz-Rivera et al. [17] in their study of scalar SF effects.

Numerical calculation of the SF:- Our numerical technique for computing the SF is described in detail in Refs. 6] (circular orbits) and [9] (eccentric orbits). The method is based on a direct integration of the linearized (hyperpoblic) set of Einstein equations in the Lorenz gauge, with a delta-function source representing the stress-energy of the moving particle. The set of $10 \mathrm{cou}-$ pled equations is decomposed into tensor-harmonic $(l, m)$ modes, and we solve for each $l, m$-mode of the (retarded) metric perturbation using finite differentiation on a characteristic mesh in 1+1-dimensions (time+radius). The values of the metric perturbation modes and their derivatives at the particle are then fed into the "mode-sum formula" [18, 19], which yields the physical (retarded) SF 
through a mode-by mode regularization. The conservative piece $F^{\alpha}$ is finally constructed using Eq. (8).

For our ISCO analysis we need the values of the SF coefficients $F_{0}^{r}, F_{1}^{r}$ and $F_{\varphi}^{1}$, evaluated at $r_{0}=6 M$. These are extracted by fitting our numerical SF data along the orbit using the analytic formulae (15) and (16). We used two independent methods to obtain the necessary data. The first is based on applying our eccentric-orbit code [9] for a sequence of slightly-eccentric geodesics approaching the ISCO. The second method (following the treatment of Diaz-Rivera et al. in the scalar-field case [17]) is based on expanding the perturbation equations about a circular orbit and solving through $O(e)$ for a sequence of circular geodesics approaching $r_{0}=6 \mathrm{M}$. In both methods, the ISCO limit is quite delicate (as the radial period tends to infinity there) and requires a careful treatment - see [9] for a detailed discussion of our numerical procedure and error analysis. Reassuringly, our two methods produce mutually consistent numerical data.

Results and discussion:-We obtained $F_{0 \mathrm{is}}^{r}=$ $0.0244666 \mu / M^{2}, \quad F_{1 \text { is }}^{r}=0.06209 \mu / M^{2}$ and $F_{\varphi \text { is }}^{1}=$ $-1.07 \mu$. Equations (18) and (20) then give, respectively,

$$
\Delta r_{\text {isco }}=-3.269 \mu, \quad \frac{\Delta \Omega_{\text {isco }}}{\Omega_{\text {isco }}}=0.4870 \mu / M .
$$

We estimate [9] the absolute numerical error in these figures as $\pm 0.003 \mu$ and $\pm 0.0006 \mu / M$, respectively. This error is controllable and can be reduced in the future simply by running our code for longer. Recall the quoted values correspond to the Lorenz gauge. Note the ISCO frequency increases under the conservative effect of the gravitational SF. This is qualitatively similar to the effect of the scalar SF acting on a particle endowed with scalar charge, which also increases $\Omega_{\text {isco }}[17]$.

It is interesting to compare the conservative shift $\Delta \Omega_{\text {isco }}$ with the frequency bandwidth of the dissipative transition across the ISCO. From the analysis of OT [11] one estimates the latter (in the Schwarzschild case) as $\Delta \Omega_{\text {diss }} / \Omega_{\text {isco }} \cong 4.3874 \eta^{2 / 5}$, where, recall, $\eta=\mu / M$. Hence, $\Delta \Omega_{\text {diss }} / \Delta \Omega_{\text {isco }} \cong 9 \eta^{-3 / 5}$, giving, for example, $\sim 35830,9000$ and 2261 for mass ratios $\eta=10^{-6}, 10^{-5}$ and $10^{-4}$, respectively. Thus, for $\eta$ in the astrophysicallyrelevant range, the conservative piece of the SF shifts the ISCO frequency by an amount much smaller than the entire bandwidth of the transition regime. This confirms what one expects based simply on the above $\eta^{-3 / 5}$ scaling, and it provides a firm justification for omitting the conservative $\mathrm{SF}$ in the $\mathrm{OT}$ analysis.

The main practical value of our result, we believe, is in that it provides an accurate strong-field benchmark to inform the development of approximate methods, such as PN. A particularly promising PN treatment of the small- $\eta$ inspiral problem is based on the Effective One Body (EOB) approach [20], with a recent study [21] convincingly demonstrating the effectiveness of EOB in describing the dissipative part of the strong-field dynamics. It would be interesting to test the performance of the conservative EOB dynamics against the results obtained here. We also envisage that our results could help inform the refinement of approximate "Kludge" models for gravitational waveforms from astrophysical inspirals [22].

We acknowledge support from PPARC/STFC through grant number PP/D001110/1. NS also acknowledges support from Monbukagaku-sho Grant-in-Aid for the global COE program "The Next Generation of Physics, Spun from Universality and Emergence".
[1] F. Pretorius, Phys. Rev. Lett. 95, 121101 (2005); M. Campanelli et al., Phys. Rev. Lett. 96, 111101 (2006); J. G. Baker et al., Phys. Rev. Lett. 96, 111102 (2006).

[2] L. Barack and C. Cutler, Phys. Rev. D 69, 082005 (2004).

[3] J. A. Gonzalez, U. Sperhake and B. Brugmann, arXiv:0811.3952 [gr-qc].

[4] Y. Mino, M. Sasaki and T. Tanaka, Phys. Rev. D 55, 3457 (1997); T. C. Quinn and R. M. Wald, Phys. Rev. D 56, 3381 (1997); E. Poisson, Living Rev. Relativity, 7, 6 (2004); S. E. Gralla and R. M. Wald, Class. Quant. Grav. 25, 205009 (2008).

[5] L. Barack and C. O. Lousto, Phys. Rev. D 66, 061502 (2002).

[6] L. Barack and N. Sago, Phys. Rev. D 75, 064021 (2007).

[7] S. Detweiler, Phys. Rev. D 77, 124026 (2008).

[8] N. Sago, L. Barack and S. Detweiler, Phys. Rev. D 78, 124024 (2008).

[9] L. Barack and N. Sago, in preperation.

[10] T. Damour, P. Jaranowski and G. Schaefer, Phys. Rev. D 62, 084011 (2000); L. Blanchet and B. R. Iyer, Class. Quant. Grav. 20, 755 (2003).

[11] A. Ori and K. S. Thorne, Phys. Rev. D 62, 124022 (2000).

[12] R. O'Shaughnessy, Phys. Rev. D 67, 044004 (2003).
[13] P. A. Sundararajan, Phys. Rev. D 77, 124050 (2008).

[14] See, e.g., Eq. (2.80) in T. Hinderer and E. E. Flanagan, Phys. Rev. D 78, 064028 (2008), which states a more general version of this symmetry. The idea to exploit the symmetries of Kerr geodesics in SF calculations is originally due to Y. Mino, Phys. Rev. D 67, 084027 (2003).

[15] L. Barack and A. Ori, Phys. Rev. D 64, 124003 (2001).

[16] More precisely, $\Omega$ is invariant under any $O(\mu)$ gauge displacement $\xi^{\alpha}$ satisfying $\left(\partial_{t}+\Omega \partial_{\varphi}\right) \xi^{\alpha}=0$ as well as $\xi^{\theta}=0$ along the orbit; see Ref. [8].

[17] L. M. Diaz-Rivera, E. Messaritaki, B. F. Whiting and S. Detweiler, Phys. Rev. D 70, 124018 (2004).

[18] L. Barack and A. Ori, Phys. Rev. D 61, 061502R (2000).

[19] L. Barack, Y. Mino, H. Nakano, A. Ori and M. Sasaki, Phys. Rev. Lett. 88, 091101 (2002).

[20] A. Buonanno and T. Damour, Phys. Rev. D 59 (1999) 084006.

[21] T. Damour and A. Nagar, Phys. Rev. D 76, 064028 (2007).

[22] S. Babak, H. Fang, J. R. Gair, K. Glampedakis and S. A. Hughes, Phys. Rev. D 75, 024005 (2007). 